\title{
Updates in Pediatric Hospital Medicine: Six Practical Ways to Improve the Care of Hospitalized Children
}

\author{
Courtney L Edgar-Zarate, MD ${ }^{1, *}$; Christopher J Russo, MD²
}

'Department of Internal Medicine and Pediatrics, University of Arkansas for Medical Sciences, Little Rock, Arkansas; ${ }^{2}$ Centra Medical Group, Lynchburg, Virginia.

BACKGROUND: As pediatric hospital medicine continues to grow, it is important to keep abreast of the current literature. This article provides a summary of six of the most impactful articles published in 2018.

METHODS: The authors reviewed articles published between January 2018 and December 2018 for the 2019 Society of Hospital Medicine national conference presentation of Top Articles in Pediatric Hospital Medicine, where the top 10 articles of 2018 were presented. Six of the 10 articles are highlighted in this review based on article quality and their applicability to change practices in the hospital setting or prompt further research.

RESULTS: Key findings from the articles include: multiple interventions aimed at providers can improve compliance with bronchiolitis guidelines; a developed calculator can improve testing for urinary tract infections in children aged 2-24 months; nonmedical costs of hospitalizations are underappreciated and disproportionately affect those with a lower socioeconomic status; a progress note template in an electronic health record can lead to higher quality and shorter notes; for febrile infants aged 60 days and younger, most blood and cerebrospinal fluid culture pathogens can be identified within 24 hours and nearly all by 36 hours; and the development of a high-value care tool can help to bring concepts of high-value care into family-centered rounds.

CONCLUSION: The six selected articles highlight findings pertinent to pediatric hospital medicine. Journal of Hospital Medicine 2019;14:436-440. Published online first June 12, 2019. (@ 2019 Society of Hospital Medicine

\section{T} he field of pediatric hospital medicine has seen tremendous growth in scholarship in the past decade. To obtain a wide view of advancements in the field from the current literature, the authors selected $18 \mathrm{En}$ glish-language journals (Table 1) across four domains believed to be relevant to the practice of pediatric hospital medicine, including hospital medicine, pediatrics, emergency care, and medical education. The median Hirsch index (h-index) of the selected journals was 131. A goal of 10, a number that could maximally benefit consumers of the finished product, was set as the final number of articles to be selected.

Guiding principles for the initial selection included novelty of hypotheses, study design, significance of results, and likelihood to change pediatric hospital medicine practice from both the community and academic hospital perspectives. Journals were assigned randomly to each author for review and assignments were switched after six months to limit potential bias in coverage. A three-stage review process was employed. The authors initially independently reviewed titles and abstracts from 13,296 articles published between January 2018 and December 2018 and rated them according to their likelihood

*Corresponding Author: Courtney L Edgar-Zarate, MD; E-mail: cledgarzarate@uams.edu; Telephone: 501-364-4361

Received: February 11, 2019; Revised: April 3, 2019; Accepted: April 7, 2019

(c) 2019 Society of Hospital Medicine DOI 10.12788/jhm.3226 to be included in the final set of 10 articles and their broad applicability to pediatric hospital medicine. This resulted in 99 studies that were selected for further review. Next, the authors were assigned a subset of the 99 articles for further review;

\section{TABLE 1. List of Journals Reviewed}

Academic Medicine

Academic Pediatrics

Annals of Emergency Medicine

Archives of Disease in Childhood

British Medical Journal

British Medical Journal - Paediatrics

Hospital Pediatrics

Journal of Graduate Medical Education

Journal of Hospital Medicine

Journal of Pediatrics

Journal of the American Medical Association

Journal of the American Medical Association Pediatrics

The Lancet

The Lancet Child and Adolescent Health

New England Journal of Medicine

Pediatric Emergency Care

Pediatric Infectious Disease Journal

Pediatrics 
1. Novel interventions, such as a real-time data dashboard of provider performance, can improve compliance with clinical practice guidelines for bronchiolitis.

2. UTICalc is a calculator that evaluates the pre- and posttest probability of urinary tract infections in children aged 2-24 months with excellent sensitivity and specificity.

3. Nonmedical costs of hospitalizations can cause significant financial burdens for families, with a median household losing $45 \%$ of their daily household income during a hospitalization.

This loss is more significant for those already facing social and financial hardships.

4. An electronic health record progress note template using best-practice guidelines can lead to higher quality, shorter, and earlier-completed notes.

5. For febrile infants aged $\leq 60$ days with bacteremia or meningitis, $88 \%-89 \%$ of the pathogens present are detected by 24 hours, and $95 \%$ are detected by 36 hours; well-appearing infants could be considered for discharge after 24 hours.

6. A newly developed, validated rounding tool focusing on high-value care items can be incorporated into family-centered rounds.

each author rated the articles independently based on their likelihood of inclusion in the final 10-article set. At this stage, 75 articles were excluded. Finally, all remaining 24 articles were reviewed independently and in depth by both authors.

Ten articles were selected by consensus formation, and the authors presented their findings at the 2019 Society for Hospital Medicine annual meeting. From these 10 articles, six were determined to be most impactful to current practice; these articles are presented below. After discussing the study background, an overview, key results, limitations of the study, important findings (Table 2), and implications for practice and future research are presented.

\section{SELECTED PUBLICATIONS}

Interventions to Reduce Over-Utilized Tests and Treatments in Bronchiolitis. Tyler A, et al. Pediatrics. 2018;141(6):e20170485. ${ }^{1}$

\section{Background}

The American Academy of Pediatrics (AAP) published clinical practice guidelines (CPG) for bronchiolitis in 2014. ${ }^{2}$ However, unnecessary tests and interventions continue to be ordered and used on children with bronchiolitis that are not recommended by the guidelines. In this quality improvement project, the authors sought to increase compliance with the AAP CPG for bronchiolitis by reducing chest x-rays (CXR) to $<20 \%$, respiratory viral testing (RVT) to $<15 \%$, and use of bronchodilators to $<20 \%$.

\section{Study Overview and Results}

This project took place at a free-standing children's hospital and included urgent care locations. Authors obtained pre-intervention data through two bronchiolitis seasons in 2013 and 2014 for patients aged 1-23 months with a primary or secondary diagnosis of bronchiolitis and who did not require admission to the Intensive Care Unit (ICU). The intervention period was from December 2015 to April 2016. All sites simultaneously implemented their interventions, which included education of care team members and families, updated order sets, and electronic health record (EHR)-generated e-mails that provided data looking at peer ranking statistics for each intervention, CXR, RVT, and bronchodilator usage. A data dashboard was created to display real-time utilization of the studied interven- tions. Providers were also asked to sign a pledge that they would reduce unnecessary testing and treatment. As balancing measures, the numbers of patients presenting to the Emergency Room (ER) or readmitted within seven days of an ED visit or admission for bronchiolitis were tracked; patients who required ICU levels of care during their first admission or on readmission were also tracked. Statistically significant decreases in CXR ordering from $39.5 \%$ to $27.2 \%$, RVT ordering from $31.9 \%$ to $26.3 \%$, and any bronchodilator usage from $34.2 \%$ to $21.5 \%$ were noted. No difference pre- and postintervention in patients readmitted to the ICU was found, and length of stay (LOS) between groups was not statistically significant.

\section{Limitations}

As all interventions were initiated simultaneously, identifying which individual or subset of interventions was responsible for changing provider behavior was impossible. More patients postintervention were admitted under observation status and under a milder All Patient Refined Diagnosis Related Groups (APR DRGs) severity index, which may indicate a less-sick cohort of patients in this group. Since the LOS and number of patients readmitted to the ICU were similar in both groups, it is unlikely that the postintervention group represented a lesssick cohort.

\section{Important Findings and Implications}

This Ql project highlighted novel ways to implement and emphasize the importance of compliance to CPG. A provider pledge may be helpful in reinforcing to all providers the idea that the institution is committed to guideline implementation. Comparing individual provider data and having a real-time dashboard with group performance can help reinforce goals and progress toward them at the group, site, and individual patient population levels.

\section{Development and Validation of a Calculator for} Estimating the Probability of Urinary Tract Infection in Young Febrile Children. Shaikh N, et al. JAMA Pediatrics. 2018;172(6):550-556. ${ }^{3}$

\section{Background}

The prevalence of urinary tract infections (UTIs) in children under 2 years of age that present to the emergency department 
(ED) with fever is about 7\%. ${ }^{4}$ After clinical examination, providers obtaining a urinalysis must then determine if empirical antibiotics are warranted for a suspected UTI. This study describes the development of a novel calculator, UTICalc that estimates the pretest probability of a UTI based on clinical findings and the posttest probability of a UTI based on laboratory results.

\section{Study Overview and Results}

This study features a single-center, nested, case-control design that looked retrospectively at 542 children aged 2-24 months who presented to the ED from January 2007 to April 2013 with fever and had a catheterized urinalysis obtained. Patients were then matched with randomly selected children without a UTI to create a training database. Five models using different variables were developed, including one with only clinical characteristics and four that combined clinical characteristics with differing laboratory values. The area under the curve of the "clinical model" was 0.80 , while those of the remaining four models ranged from 0.97 to 0.98 . The clinical model showed a sensitivity of $95 \%$ and specificity of $35 \%$ in the training database, while the four other models showed sensitivities ranging from $93 \%$ to $96 \%$ and specificities ranging from $91 \%$ to $93 \%$. The models were then validated using a cohort of children aged 2-24 months who presented to the ED with fever from July 2015 to December 2016; the UTI prevalence in this cohort was $7.8 \%$. Finally, using a hypothetical cohort of 1,000 children being evaluated for a UTI, the authors showed that UTICalc reduced the numbers of urine samples obtained by $8.1 \%$ and missed UTIs from 3 to 0 compared with following AAP guidelines. ${ }^{5}$

\section{Limitations}

The training database was created retrospectively at a single institution and is subject to local practice patterns. The proposed calculator creates an algorithm that is meant to be used in a setting where the pretest probability for a UTI is reasonably high based on criteria from the AAP UTI guidelines.

\section{Important Findings and Implications}

UTICalc could be a great tool for providers to guide testing for UTIs in children aged 2-24 months presenting with a fever. Given further study at multiple sites and settings, including outpatient clinics, UTICalc could have significant implications for reducing unnecessary testing and treatment in febrile children.

\section{Lost Earnings and Nonmedical Expenses of Pedi-} atric Hospitalizations. Chang LV, et al. Pediatrics. 2018;142(3):e20180195.6

\section{Background}

Although medical expenses related to hospitalization can be significant for many families, nonmedical costs, such as transportation, parking, meals, and lost earnings from missed days at work, are also important to consider. These hardships can lead to challenges in postdischarge follow-up and adherence to discharge instructions, both of which lead to hospital readmissions. This article presents a cross-sectional analysis at a large, free-standing children's hospital that participated in the
Hospital-to-Home Outcomes Study (H2O). The authors sought to determine whether families with more financial or social hardships are affected disproportionately by nonmedical costs related to hospitalizations.

\section{Study Overview and Results}

A total of 1,372 children were included and children with lengths of stay $>13$ days were excluded. Face-to-face parental surveys were conducted and included questions on parental education, employment status, sick leave flexibility, and measures of financial and social hardship. The study authors calculated a total cost burden (TCB) based on nonmedical costs estimated at the time of the survey, including lost wages and expenses during the hospitalization. A daily cost burden (DCB) based on length of hospital stay and daily cost burden as a percentage of daily income (DCBi) were also calculated. The median TCB was $\$ 112.80$, and the median DCB was $\$ 51.40$. The median DCBi showed that the median household had $45 \%$ of their daily income depleted by nonmedical expenses related to their hospitalization. Those who reported more financial or social hardships had a higher median $\mathrm{DCBi}$; if $\geq 3$ financial hardships were reported, $86 \%$ of the daily household income was depleted.

\section{Limitations}

The study was conducted at a single institution with a number of existing support systems in place to help unburden families of hospitalized children. Non-English-speaking families were excluded. A face-to-face survey may have influenced parental responses regarding social and financial hardships.

\section{Important Findings and Implications}

Nonmedical costs of hospitalized children can be quantified and disproportionately affect those experiencing financial and social hardships. Hospitalists should be aware of these findings and find ways within their hospital systems to provide support for families both during and after hospitalizations.

\section{A Prescription for Note Bloat: An Effective Progress Note Template. Kahn D, et al. Journal of Hospital Medicine. 2018;13(6):378-382.7 \\ Background}

Although electronic health records (EHRs) have improved the speed and legibility of documentation, the harm of "note bloat," defined as multiple pages of nonessential information which leaves key aspects buried or lost, is prevalent. In this prospective, quality improvement study across four internal medicine residency programs, the authors investigated a bundled intervention consisting of didactic teaching and an electronic progress note template on note quality, length, and timeliness.

\section{Study overview and results}

Notes pre- and postintervention were graded using a tool that considered the general impression of the note, its score on the validated Physician Documentation Quality Instrument (PDQI9), ${ }^{8}$ and a questionnaire based on the Accreditation Council for 
Graduate Medical Education competency note checklist.9 Analyzing 200 preintervention and 199 postintervention notes, significant improvement was seen in general impression scores, all PDQ1-9 domains, and 6 of 13 note competency questionnaire items. The mean number of lines in the note decreased by $25 \%$, and the mean completion time when the note was signed was 1 hour and 15 minutes earlier. The greatest impact on shortening notes involved a reduction in the auto-population of laboratory and imaging studies.

\section{Limitations}

The study was unblinded. The authors attempted to minimize bias with an objective questionnaire and employed multiple graders per note; however, poor interrater reliability was obtained. Postintervention, $70 \%$ of all residents used the template. At one of the four institutions, evidence of note quality improvement despite low template use was found. At another institution, no improvement in note quality was reported despite relatively high template uptake. Local culture and institutional buy-in may be factors affecting these results. In addition, pre- and postintervention notes were examined in the same academic year; thus, the effects seen may be due, in part, to resident maturation. Generalizability to nonacademic institutions and the durability of the intervention are additional concerns.

\section{Important Findings and Implications}

Resident education on documentation and an EHR progress note template incorporating best practices can effectively combat "note bloat" and lead to higher quality and shorter notes that are completed earlier in the day. This solution has significant implications for improving transitions of care, handoffs, and patient safety.

\section{Time to Pathogen Detection for Non-III Versus} Ill-Appearing Infants $\leq 60$ Days Old with Bacteremia and Meningitis. Aronson PL, et al. Hospital Pediatrics 2018;8(7):379-384. ${ }^{10}$

\section{Background}

The routine evaluation of febrile infants aged $\leq 60$ days old often involves blood and cerebrospinal (CSF) fluid evaluations, and many infants are hospitalized while waiting for culture results. A previous study of febrile infants showed that $91 \%$ of the pathogenic organisms could be identified on blood culture within 24 hours and that $96 \%$ could be identified within 36 hours; $81 \%$ of the bacterial pathogens present were detected on CSF culture within 36 hours. ${ }^{11}$

\section{Study Overview and Results}

In this large, multicenter study of infants presenting to the Emergency Departments (EDs) of 10 children's hospitals over a five-year study period, the authors investigated the time to pathogen detection in blood and CSF for infants aged $\leq 60$ days with bacteremia and/or bacterial meningitis; whether the time to detection differed for non-ill and ill infants was also examined. III- versus non-ill-appearance was determined by a medical record review of the physical exam looking for one of 13 key words (eg, "ill-appearing," "toxic," "lethargic," etc.). A total of 381 infants were included. Overall, $88 \%$ of the pathogens present were detected in blood culture within 24 hours and $95 \%$ were detected within 36 hours. In CSF, $89 \%$ of the pathogens present were detected within 24 hours, and $95 \%$ were detected within 36 hours. In infants with bacteremia who were non-ill-appearing, $85 \%$ of the blood pathogens were detected within 24 hours.

\section{Limitations}

The median time to detection for blood culture pathogens for ill-appearing versus non-ill-appearing infants was shorter by just one hour, but $15 \%$ of the non-ill infants had a positive blood culture after 24 hours. However, the prevalence of bacteremia and meningitis in non-ill-appearing infants is likely low; the authors did not report the total number of febrile infants evaluated by EDs in the study.

\section{Important Findings and Implications}

Most positive blood and/or CSF cultures for infants aged $\leq 60$ days will yield results by 24 hours; $95 \%$ of the pathogens present could be detected within 36 hours. Sending a non-ill-appearing febrile infant home at 24 hours may miss $15 \%$ of the instances of bacteremia, but the overall low prevalence of invasive bacterial infection in infants should be considered.

The High-Value Care Rounding Tool: Development and Validity Evidence. McDaniel CE, et al. Academic Medicine. 2018;93(2):199-206.12

\section{Background}

Providing high-value care (HVC) to patients is a struggle for physicians and healthcare systems. Although physicians teaching trainees HVC practices could be an effective way to increase cost-conscious care, the best practices for teaching HVC remain unknown. To fill this gap, the authors developed a tool to measure the frequency and content of observable HVC teaching and evaluated the validity of the tool within a pediatric inpatient setting.

\section{Study Overview and Results}

The HVC rounding tool was developed through several phases from conception to validation. The research group used a modified Delphi method to construct the tool using a consensus building process based on opinions from content experts in the field of HVC, from a variety of specialties, experience levels, and geographic areas of the United States. Each item of the HVC instrument was rated by these experts, and, from their evaluations and surveys, an 11-item HVC tool was constructed. A pilot of the tool was performed to establish internal validity and interrater reliability based on observations of 148 patient encounters. From this process, a final 10-item HVC rounding tool emerged, including domains in quality, cost, and patient values. A few items included giving positive feedback for not doing an unnecessary test, discussing whether a patient needs to stay inpatient or meets discharge criteria, and customizing a care plan to align with family values and goals. The final iteration of the tool had 
no rater disagreements within the quality and patient values domain and only one disagreement within the cost domain.

\section{Limitations}

This tool was validated at a single pediatric institution, and, thus, the generalizability of the tool has not been established. The authors note that the Delphi panelists used for the construction of the tool were from a medical subspecialty background and not surgical backgrounds, which limits its applicability from a surgical perspective. The tool does not allow for differentiation between lengthy discussions or brief comments presented during rounds.

\section{Important Findings and Implications}

The HVC rounding tool is both innovative and timely. Pediatric hospitalists are leaders in family-centered care, and this tool allows assessment of whether important concepts of high-value care are discussed at the bedside. A multisite educational study using this tool would be welcome.

Disclosures: The authors have no conflicts of interest to declare.

\section{References}

1. Tyler A, Krack P, Bakel LA, et al. Interventions to reduce over-utilized tests and treatments in bronchiolitis. Pediatrics. 2018;141(6):e20170485. doi: 10.1542/ peds.2017-0485.

2. Ralston SL, Lieberthal AS, Meissner HC, et al. Clinical practice guideline: the diagnosis, management, and prevention of bronchiolitis. Pediatrics. 2014;134(5):e1474-502. doi: 10.1542/peds.2014-2742.

3. Shaikh N, Hoberman A, Hum SW, et al. Development and validation of a calculator for estimating the probability of urinary tract infection in young febrile children. JAMA Pediatr. 2018;172(6):550-556. doi:10.1001/jamapediatrics.2018.0217.

4. Shaikh N, Morone NE, Bost JE, Farrell MH. Prevalence of urinary tract infections in childhood: a meta-analysis. Pediatr Infect Dis J. 2008;27(4):302-8. doi: 10.1097/INF.0b013e31815e4122.

5. Roberts KB. Urinary tract infection: clinical practice guideline for the diagnosis and management of the initial UTI in febrile infants and children 2 to 24 months. Pediatrics. 2011;128(3):595-610. doi: 10.1542/peds.2011-1330.

6. Chang LV, Shah AN, Hoefgen ER, et al. Lost earnings and nonmedical expenses of pediatric hospitalizations. Pediatrics. 2018;142(3):e20180195. doi: 10.1542/peds.2018-0195.

7. Kahn D, Stewart E, Duncan M, et al. A prescription for note bloat: an effective progress note template. J Hosp Med. 2018;13(6):378-382. doi: 10.12788/ jhm.2898.

8. Stetson PD, Bakken S, Wrenn JO, Siegler EL. Assessing electronic note quality using the Physician Documentation Quality Instrument (PDQI-9). Appl Clin Inform. 2012;3(2):164-174. doi: 10.4338/ACI-2011-11-RA-0070.

9. Aylor M, Campbell EM, Winter C, Phillipi CA. Resident notes in an electronic health record: a mixed-methods study using a standardized intervention with qualitative analysis. Clin Pediatr (Phila). 2016;6(3):257-262. doi: 10.1177/0009922816658651.

10. Aronson PL, Wang ME, Nigrovic $L E$, et al. Time to pathogen detection for non-ill versus ill-appearing infants $\leq 60$ days old with bacteremia and meningitis. Hosp Pediatr. 2018;8(7):379-384. doi: 10.1542/hpeds.2018-0002.

11. Biondi EA, Mischler M, Jerardi KE, et al. Blood culture time to positivity in febrile infants with bacteremia. JAMA Pediatr. 2014;168(9):844-849. doi: 10.1001/jamapediatrics.2014.895.

12. McDaniel CE, White AA, Bradford MC, et al. The high-value care rounding tool: development and validity evidence. Acad Med. 2018;93(2):199-206. doi: 10.1097/ACM.0000000000001873. 